\title{
Polysomnographic Evaluation of OSA in Patients of Bronchial Asthma
}

\author{
Dar Mohd Yousoof ${ }^{* 1}$, Jamal Mohammad ${ }^{2}$, Mir Shahnawaz ${ }^{2}$, Shah Naveed Nazir ${ }^{3}$, Dar Khursheed ${ }^{3}$, Syed Suriya ${ }^{4}$ \\ ${ }^{1}$ Lecturer, Chest Diseases Hospital, Govt. Medical College Srinagar \\ ${ }^{2}$ Junior Resident, Chest Diseases Hospital, Govt. Medical College Srinagar \\ ${ }^{3}$ Professor, Chest Diseases Hospital, Govt. Medical College Srinagar \\ ${ }^{4}$ Associate Professor, Chest Diseases Hospital, Govt. Medical College Srinagar
}

*Corresponding author: Mohd Yousoof Dar, MD, DM; yousufhumi@gmail.com

Received: 19 November 2020;

Accepted: 06 December 2020;

Published: 12 December 2020

\begin{abstract}
Introduction: Bronchial asthma a common non-communicable disease affecting more than 300 million people world-wide. Compared to general population quality of sleep is poor in asthma patients due to various factors like poor control of asthma, co-morbidities like allergic rhinitis and oesophageal reflex disease but little is known about the potential role of obstructive sleep apnoea. Aims and objectives: This study was conducted is to evaluate the association between Asthma and risk of developing Obstructive Sleep Apnoea. Materials and methods: This was a prospective design study conducted on 137 patients of diagnosed bronchial asthma attending out-patient department of a referral chest from north India after ethical clearance. These patients underwent Berlin score assessment first and later level-1 polysomnography to evaluate for OSA. Results: prevalence of PSG documented OSA observed was $53.3 \%$ which is significantly higher than the general population and there was significant association with increasing age as $54.7 \%$ of patients were in middle aged population (40-60 years) and obesity with $67.1 \%$ patients having OSA with BMI $>30$ compared to $37.5 \%$ prevalence only with BMI $<30$, as well as with severity of asthma. Besides OSA was seen in $91.9 \%$ of Berlin high risk group patients, while it was found in only $21.3 \%$ of Berlin low-risk group. Conclusion: prevalence of OSA in asthma patients is significantly higher as compared to general population and there is significant positive co-relation between severity of asthma, BMI, increasing age and higher Berlin score with the overall prevalence of OSA.
\end{abstract}

Keywords: BMI- Body mass index, GINA- Global initiative for Asthma, ICS-inhaled corticosteroids OCS- oral corticosteroids, PSGpolysomnography, OSA-Obstructive Sleep Apnoea

\section{Introduction}

Bronchial asthma is a common non-communicable chronic inflammatory airway disease, characterized by bronchial hyperresponsiveness and variable airflow obstruction. It is estimated to affect more than 300 million people world-wide ${ }^{[1]}$. Health-related quality of life of asthma patients is directly related to their level of asthma control ${ }^{[2]}$, but various large community surveys have shown that this is rarely achieved in real world scenario ${ }^{[3]}$. Compared to general population, quality of sleep is poor in asthma patients due to various factors like poor control of asthma, comorbidities like allergic rhinitis and oesophageal reflex disease but little is known about the potential role of obstructive sleep apnoea ${ }^{[4]}$. Regarding co-relation of obstructive sleep apneoa (OSA) with asthma, different cross-sectional studies have documented prevalence of OSA to be significantly higher in asthma patients as compared to general population ${ }^{[5]}$. Risk of OSA appears to be almost double in asthmatic populations as shown by various studies and common positive moderators of this risk being evaluated are: asthma severity, level of asthma control, black race, obesity and female gender ${ }^{[6]}$.

\section{Aim}

Aim of our study was to evaluate the association between Asthma and risk of developing Obstructive Sleep Apnea.

\section{Materials and methods}

This was a prospective design study conducted over a period of 2 years from February 2017 to February 2019 after taking ethical clearance in a tertiary referral chest hospital of Jammu and Kashmir belonging to north India. A total of 153 patients were enrolled in this study. These patients were subjected to inclusion and exclusion criteria and 16 patients were excluded as they were not fulfilling the criteria. So a total of 137 patients of bronchial asthma diagnosed as per GINA 2016 were included in study. All the patients underwent Berlin score assessment first and then it was 
followed by level-1 Polysomnography(PSG) to evaluate for OSA . Once OSA was diagnosed it was further classified as mild, moderate, and severe OSA depending AHI values observed.

\section{Inclusion Criteria for the patient}

1. Bronchial asthma diagnosis as per GINA guidelines December 2016 with spirometry proven reversible airway obstruction.

2. Age group: $18-65 \mathrm{yrs}$.

3. Suspicion of sleep disordered breathing as per Berlin Questionnaire risk score.

4. Patients willing to give written informed consent to participate in the study.

\section{Exclusion Criteria for the patient}

1. Current smoker or history of smoking.

2. Pregnant females.

3. Any patient who had acute exacerbation in last 4 weeks or presently in exacerbation.

4. Uncooperative patient and not given consent.

5. Significant comorbidities of CLD, ESRD, decompensated heart failure.

\section{Methodology}

After taking written informed consent, all patients were subjected to detailed history taking and physical examination, Chest X-ray, Electrocardiogram (ECG), Body mass index calculation, Pulmonary function testing including spirometry with pre- and post-bronchodilation values, Berlin Questionnaire to assess for risk of presence of OSA and Overnight level I [in-lab] polysomnography to confirm OSA.

Bronchial asthma was diagnosed according to GINA guideline 2016 by performing Pulmonary Function Test and documenting airflow limitation (FEV1/FVC $<70 \%$ ) at least once during workup and positive bronchodilator reversibility test by demonstrating increase in FEV1 of $>12 \%$ and $200 \mathrm{ml}$ from baseline after inhalation of 200-400mcg of salbutamol. Pulmonary Function Test was done on PFT lab with integrated body plethysmography and diffusion system from Life Care Company. The patients were categorized as intermediate, mild, moderate and severe persistent asthma. Once bronchial asthma was diagnosed patients were accessed by Berlin Questionnaire and then taken for overnight PSG which was done with Alice- 5 model PSG lab.

Diagnosis of OSA was made as per AASM diagnostic criterion as listed in ICSD Manual, 3nd edition ${ }^{[7]}$ requiring the patient to have at least one of the following : Either symptoms of nocturnal breathing disturbances (snoring, snorting, gasping, or breathing pauses during sleep) or daytime sleepiness or fatigue that occurs despite sufficient opportunities to sleep and documenting five or more episodes of obstructive apnea or hypopnea per hour of sleep (The AHI-Apnea Hypopnea Index, calculated as the number of episodes divided by the number of hours of sleep) during overnight sleep study. OSA was further classified as mild, moderate and severe based on the basis of AHI with Mild OSA as: 5-14 events/hour, Moderate OSA as: 15-29 events/hour and Severe OSA as: > to 30 events/hour respectively.

\section{Statistical analysis}

Statistical Methods: The data analysis was done with SPSS Version 20.0 (SPSS Inc., Chicago, Illinois, USA). Continuous variables were expressed as Mean $\pm \mathrm{SD}$ and categorical variables were summarized as frequencies and percentages. Student's independent t-test was employed for comparing continuous variables and Chi-square test or Fisher's exact test, whichever appropriate, was applied for comparing categorical variables. A Pvalue of less than 0.05 was considered statistically significant.

\section{Results and observation}

A total of 137 patients of bronchial asthma were studied with mean age $47.9 \pm 12.39$, ranging from 23 to 76 years of age. Gender distribution was almost equal as $70(51.1 \%)$ patients were male and $67(48.9 \%)$ were female. BMI of study group showed that more than half were obese $(53.3 \%)$ and around $22.6 \%$ were overweight with Mean + SD for BMI was $29.8 \pm 5.14$. Regarding the severity of asthma, more than half (54.7\%) was having mild asthma and 22.6 $\%$ were having severe asthma. Results showed overall prevalence of OSA was more than $50 \%$ in the study population with 73 patients $(53.3 \%)$ having OSA and further OSA was seen more prevalent in females than in male population. [Tab. 1]

When prevalence of OSA was evaluated with regard to severity of asthma, it showed $70.9 \%(22 / 31)$ of severe asthma had OSA, while as only $44 \%$ (33 out of 75) of mild Asthma had OSA. [Tab.2] Furthermore severity of OSA in study population showed that $35.6 \%$ of patients had mild OSA, $21.9 \%$ patients had moderate OSA and $42.5 \%$ patients had severe OSA.

Prevalence of OSA showed significant correlation with BMI in study group as seen generally in normal population. Patients with BMI > 30 showed OSA prevalence of more than sixty percent $(67.1 \%)$ compared to patients whose with $\mathrm{BMI}<30$ in whom it was only $37.5 \%$ with a P-value $=<0.001$ which is statistically significant. [Tab. 3 and Fig. 1] When OSA prevalence was assessed in relation to Berlin score, it showed significant corelation with risk score. It was observed that in Berlin high risk group, OSA was seen in $91.9 \%$ of patient while it was only $21.3 \%$ in Berlin low-risk group with a P-value $=<0.001$ which is statistically significant. [Tab. 4 and Fig. 2]

Table 1: Showing gender-wise prevalence of OSA in study patients

\begin{tabular}{|l|l|l|l|l|}
\hline \multirow{2}{*}{ Gender } & \multicolumn{2}{|l|}{ OSA } & \multicolumn{2}{l|}{ No OSA } \\
\cline { 2 - 5 } & No. & \%age & No. & \%age \\
\hline Male & 30 & 42.9 & 40 & 57.1 \\
\hline Female & 43 & 64.2 & 24 & 35.8 \\
\hline Total & 73 & 53.3 & 64 & 46.7 \\
\hline
\end{tabular}

Table 2: Showing prevalence of OSA as per severity of asthma

\begin{tabular}{|l|l|l|l|l|}
\hline \multirow{2}{*}{ Severity of Asthma } & \multicolumn{2}{|l|}{ OSA } & \multicolumn{2}{l|}{ No OSA } \\
\cline { 2 - 5 } & No. & \%age & No. & \%age \\
\hline Mild Asthma & 33 & 44.0 & 42 & 56 \\
\hline Moderate Asthma & 18 & 58.0 & 13 & 42 \\
\hline Severe Asthma & 22 & 70.9 & 9 & 29.1 \\
\hline Total & 73 & 53.3 & 64 & 46.7 \\
\hline
\end{tabular}

Table 3: Showing prevalence of OSA as per BMI in study patients

\begin{tabular}{|l|l|l|l|l|}
\hline \multirow{2}{*}{ BMI } & \multicolumn{3}{|l|}{ OSA } & No OSA \\
\cline { 2 - 5 } & No. & \%age & No. & \% age \\
\hline$<30$ & 24 & 37.5 & 40 & 62.5 \\
\hline$\geq 30$ & 49 & 67.1 & 24 & 32.9 \\
\hline Total & 73 & 53.3 & 64 & 46.7 \\
\hline $\begin{array}{l}\text { Chi-square }=12.02 ; \quad P \text {-value }=\mathbf{0 . 0 0 1} \\
\text { Difference) }\end{array}$ & (Statistically & Significant \\
\hline
\end{tabular}




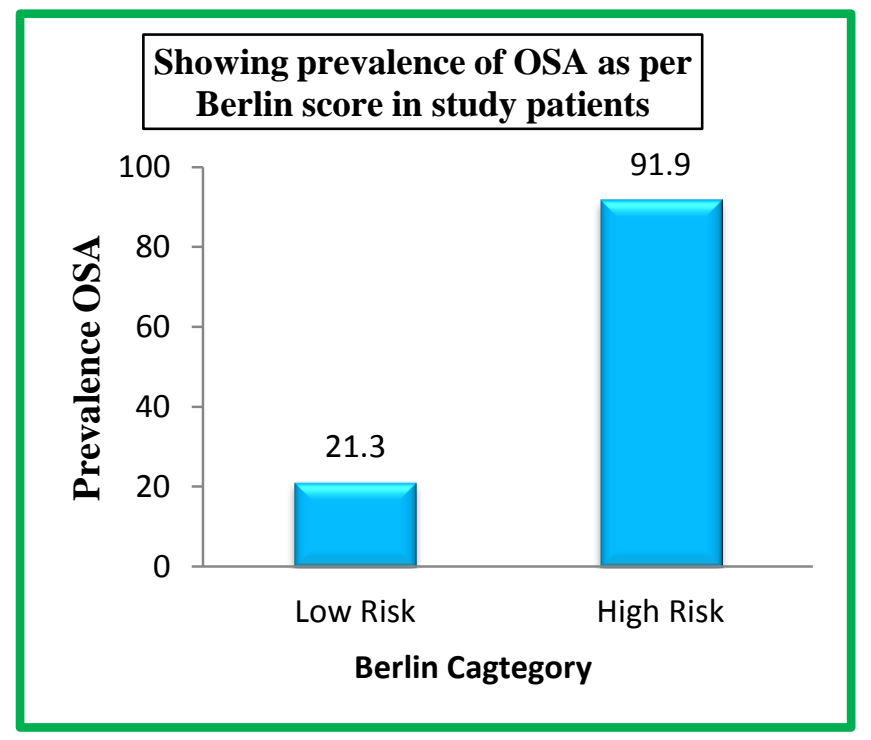

Figure 1

Table 4: Showing prevalence of OSA as per Berlin score in study patients

\begin{tabular}{|l|l|l|l|l|}
\hline \multirow{2}{*}{ Berlin Score } & \multicolumn{3}{|l|}{ OSA } & No OSA \\
\cline { 2 - 5 } & No. & \%age & No. & \%age \\
\hline Low Risk & 16 & 21.3 & 59 & 78.7 \\
\hline High Risk & 57 & 91.9 & 5 & 8.1 \\
\hline Total & 73 & 53.3 & 64 & 46.7 \\
\hline $\begin{array}{l}\text { Chi-square=67.96; P-value }<0.001 \text { (Statistically Significant } \\
\text { Difference) }\end{array}$ \\
\hline
\end{tabular}

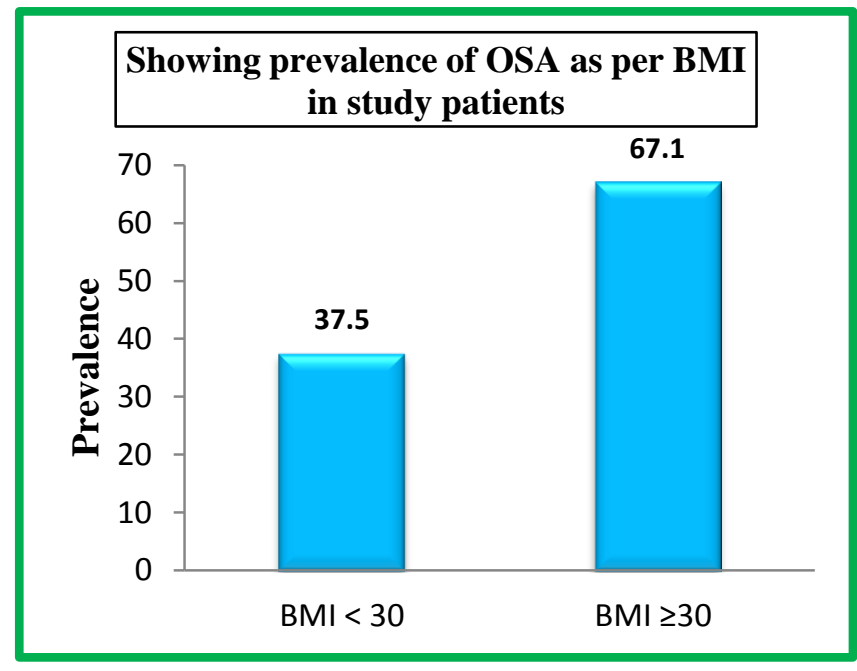

Figure 2

\section{Discussion}

World over asthma-related deaths tend to occur during the night or early morning because symptoms are generally more severe at night which is suggestive of poorly controlled underlying asthma ${ }^{[1]}$ and as many as $64-74 \%$ of asthma patients are having nocturnal symptoms ${ }^{[8]}$. The results of our study confirm this fact and show the presence of nocturnal symptoms in many of our patients. The mechanisms by which nocturnal asthma develops remain unclear and may vary from patient to patient ${ }^{[9,10]}$. One of the potential mechanisms believed is reduction in the cross-sectional surface area of upper airway due to persistent airway mucosal inflammation in poorly controlled asthma. This was demonstrated by Collett and colleagues ${ }^{[11]}$ by radiographic techniques of pharynx during a broncho-provocation test in asthma patients.
Yigla, et al. ${ }^{[12]}$ found very high prevalence of OSA (around 95\%) in asthmatics that were on long term oral corticosteroids (OCS) or requiring frequent bursts of OCS. This high prevalence of OSA is postulated to OCS effect of para-pharyngeal fat deposition and steroid myopathy on the upper airway which leads to an increase in upper airway collapsibility and hence features of OSA. In another study by Teodorescu, et al. ${ }^{[13]}$ increased risk of OSA with ICS use was observed in a dose dependent manner. Regardless of what precipitates nocturnal asthma, in turn it leads to chronic sleep fragmentation, which promotes upper airway collapsibility.

The results of our study found significant association between the age of patients and prevalence of OSA. The mean age of population having OSA in our study was $47.9 \pm 12.39$ years with $54.7 \%$ of patients being of age group of 40-60 years. Bixler, et al. [14] in their study found similar result with prevalence of OSA increasing progressively up to age of 55 years, after which it remained static. Shen, et al. ${ }^{[15]}$ in their study also found higher mean age in OSA group.

We observed OSA to be more common in females (64.2\%) compared to males $(44.9 \%)$ which is statically significant. Similar results of high female predominance of OSA were also reported by Zidan, et al. ${ }^{[16]}$ of $66.6 \%$ and by Madama, et al. ${ }^{[17]}$ of $59.2 \%$. Further OSA was more prevalent in obese patients in our study with prevalence of $67.1 \%$ in patients with BMI >30 compared to $37.5 \%$ only in patients with BMI $<30$ which is statistically significant. Similar result was reported by Zidan, et al. ${ }^{[16]}$ and Julien, et al. ${ }^{[18]}$ in their studies.

Overall prevalence of OSA in our study group was 53.3\%, which is consistent with other studies done by Janson et al, Min Kwang, et al and many others. Janson, et al. ${ }^{[19]}$ in a study of 2202 patients from 3 different European countries found OSA symptoms to be $50 \%$ more common in asthmatic group with the conclusion that net result of both diseases (OSA and asthma) will lead poor quality of sleep with its impact on day-time activity and overall poor quality of life of the patient. Min Kwang, et al. ${ }^{[20]}$ in their study diagnosed OSA in $66.5 \%$ of patients with underlying bronchial asthma and concluded that moderate to severe asthma has strong correlations with OSA in addition to of age and male gender.

Furthermore when we evaluated prevalence of OSA with regard to severity of asthma, we found $70.9 \%$ (22/31) of severe asthma having OSA, while as only $44 \%$ (33 out of 75) of mild Asthma had OSA. Yigla, et al. ${ }^{[21]}$ in a small study of 22 patients with severe uncontrolled asthma on long term oral steroids observed OSA in 21 out of the 22 patients. In another study by Julien, et al. OSA was found in $50 \%$ of severe asthmatics compared to $23 \%$ only in patients with moderate asthma. The study concluded that there is positive correlation between the severity of asthma and the prevalence of OSA. Our study showed OSA to be more prevalent as compared to study by Julien, et al. for which possible reasons we believe are: a) overall prevalence of OSA was high in our study group and b) majority of our study patient population was having BMI $>30$.

Lastly our study results reviled significant co-relation of Berlin risk score with prevalence of OSA, as $91.9 \%$ were having PSG documented OSA in Berlin high-risk group compared to only $21.3 \%$ in Berlin low-risk group. We observed sensitivity and specificity of Berlin Questionnaire as $78.08 \%$ and $92.19 \%$ respectively and accuracy of around $84 \%$. Our study is one of few studies which have validated results of Berlin Questionnaire by a poly-somnographic study (Level-1). Netzer, et al. ${ }^{[22]}$ validated the Berlin Questionnaire in general population from a primary health care setting and he observed sensitivity and specificity of $86 \%$ and 
$77 \%$ respectively. Our study showed overall higher specificity of Berlin Questionnaire possibly due to differences in characteristics of study population and overall high prevalence of OSA in our study group.

\section{Conclusion}

We thus observe that prevalence of OSA in asthma patients is significantly higher as compared to general population and there is significant positive co-relation between severity of asthma, higher BMI, increasing age and higher Berlin score with prevalence of OSA. It is already established that diagnosing OSA and treating it early will improve quality of sleep and also overall quality of life.

We thus recommend routine screening for OSA in middle aged patients with moderate to severe asthma and Berlin Questionnaires is a good screening tool for this before proceeding for polysomnographic evaluation.

\section{Limitations of our study}

Obese patients were not excluded from this study, so obesity is confounding factor in this study as obesity itself is well established independent risk factor for OSA.

\section{Conflict of interest}

None

\section{Bibliography}

[1] Global strategy for asthma management and prevention (update), GINA 2016.

[2] Bateman ED, Bousquet J, Keech ML, Busse WW, Clark TJ, Pedersen SE. The correlation between asthma control and health status: the GOAL study. Eur Respir. J 2007; 29 Suppl 1: 56-62.

[3] Lai CK, De Guia TS, Kim YY, Kuo SH, Mukhopadhyay A, Soriano JB et al: Asthma control in the Asia-Pacific region: the Asthma Insights and Reality in Asia-Pacific Study. J Allergy Clin. Immunol 2003;111 Suppl 2: 263268

[4] Teodorescu M, Polomis DA, Gangnon RE, Fedie JE, Consens FB, Chervin RD et al. Asthma control and its relationship with Obstructive Sleep Apnea (OSA) in older adults. Sleep Disord. 2013; 2013:251567. Epub 2013 Nov 6.

[5] Larsson LG, Lindberg A, Franklin KA, Lundback B. Symptoms related to obstructive sleep apnoea are common in subjects with asthma, chronic bronchitis and rhinitis in a general population. Respir Med 2001; 95 (Suppl 5):423-429.

[6] Teodorescu M, Polomis DA, Hall SV, Teodorescu MD, Gangnon RE, Peterson AG, et al. Association of obstructive sleep apnea risk with asthma control in adults. Chest 2010; 138(Suppl 3): 543-550.

[7] Darien, IL. International Classification of Sleep Disorders 3rd ed. American Academy of Sleep Medicine; 2014.
[8] Jonathan P, Parsons, John G and Mastronarde. Nocturnal Asthma and Obstructive Sleep Apnea. Clinical Asthma. 2008; (Chapter 41) :367-373 Available online 22 May 2009.

[9] Kasasbeh A, Kasasbeh E, Krishnaswamy G. Potential mechanisms connecting asthma, esophageal reflux, and obesity/sleep apnea complex - a hypothetical review. Sleep Med Rev 2007 ;11(Suppl 1):47-58.

[10] Calhoun WJ. Nocturnal asthma. 2003 ;123( Suppl 3):399S-405S

[11] Collett PW, Brancatisano AP, Engel LA. Upper airway dimensions and movements in bronchial asthma. Am Rev Respir Dis 1986;133(6):1143.

[12] Yigla M, Tov N, Solomonov A, Rubin AHE, Harlev D. Difficult-to-control asthma and obstructive sleep apnea. J Asthma 2003;40(8):865-71.

[13] Teodorescu M, Consens FB, Bria WF, Coffey MJ, McMorris MS, Weatherwax KJ, et al. Predictors of habitual snoring and obstructive sleep apnea risk in patients with asthma. Chest 2009;135 (5) :1125-32.

[14] Bixler EO, Vgontzas AN, Ten Have T, Tyson K, Kales A. Effects of age on sleep apnea in men. I. Prevalence and severity. Am J Respir Crit Care Med 1998 ;157(1):144-48

[15] Shen TC, Lin CL, Wei CC, Chen CH, Tu CY, Hsia TC ,et al. Risk of Obstructive Sleep Apnea in Adult Patients with Asthma: A Population-Based Cohort Study in Taiwan. PLOS ONE 2019; 14(7): e0219612

[16] Zidan M, Daabis R, Gharraf H. Overlap of obstructive sleep apnea and bronchial asthma: Effect on asthma control. Egyptian J Chest Dis Tuberc 2015; 64(2):42530.

[17] Madama D, Silva A, Matos MJ. Overlap syndrome Asthma and obstructive sleep apnea. Rev Port Pneumo 2016; 22(1):6-10.

[18] Julien JY, Martin JG, Ernst P, Naor N, Olha A, Kimoff RJ, et al. Prevalence of obstructive sleep apnea-hypopnea in severe versus moderate asthma. J Allergy Clin Immunol. 2009;124 (2) :371-67.

[19] Janson C, backer WD, Gislason T, Plaschke P, Björnsson E, Hetta $J$, et al. Increased prevalence of sleep disturbances and daytime sleepiness in subjects with bronchial asthma: a population study of young adults in three European countries, Eur. Respir. J 1996; 10: 2132 38.

[20] Byun MK, Park SC, Chang YS, Kim YS, Kim SK, Kim $\mathrm{HJ}$, et al. Associations of moderate to severe asthma with obstructive sleep apnea. Yonsei Med J. 2013; 54(4) : 942-48.

[21] Yigla M, Tov N, Solomonov A, Rubin AHE, Harlev D. Difficult-to-control asthma and obstructive sleep apnea. J Asthma 2003; 40:865-71.

[22] Netzer NC, Stoohs RA, Netzer CM, Clark K, Strohl KP. Using the Berlin questionnaire to identify patients at risk for the sleep apnea syndrome. Ann Intern Med. 1999;131 (7):485-491. 